\title{
Columna cervical reumática
}

\author{
Artrite reumatoide da coluna cervical \\ Rheumatoid arthritis of the cervical spine
}

\author{
Nicolás Macchiavello Cornejo ${ }^{1}$ \\ Marcos Ganga Villagrán' \\ Manuel Pellegrini Pucci \\ Ronald Schulz Ibaceta'
}

\section{RESUMEN}

La artritis reumatoidea (AR) afecta a millones de personas en el mundo. Hasta un $86 \%$ de los pacientes presenta alteraciones a nivel cervical. Los patrones de inestabilidad cervical asociados a la artritis reumatoidea son: inestabilidad atlanto-axial, impactación atlanto-axial y subluxación subaxial. Una vez que aparecen alteraciones neurológicas su progresión puede ser rápida y llevar incluso a la muerte. El tratamiento de la AR es fundamentalmente médico. Los medicamentos y esquemas actuales podrían prevenir o retardar la aparición de alteraciones a nivel atlanto-axial. El examen clínico y radiológico periódico permite detectar a los pacientes que ya presentan compromiso neurológico, o a los que están en riesgo de desarrollarlo, en cuyos casos debe considerarse la cirugía como opción terapéutica. La recuperación neurológica post-quirúrgica depende del nivel de compromiso neurológico previo, lo que refuerza la importancia de la detección y derivación precoz de los pacientes en riesgo. El tratamiento quirúrgico debe llevarse a cabo en centros especializados, por grupos multidisciplinarios. Esto, junto con la intervención temprana, ayudaría a reducir las complicaciones peri-operatorias.

DESCRIPTORES: Artritis reumatoide/ cirugía; Vértebras cervicales/ patología; Enfermedades de la columna vertebral

\section{RESUMO}

A Artrite Reumatóidea (AR) afeta milhões de pessoas no mundo. Até $86 \%$ dos pacientes apresentam alterações a nível cervical. Os padrões de instabilidade cervical associados à AR são: instabilidade atlanto-axial, impacto atlanto-axial e sub-luxação sub-axial. Uma vez que aparecem as alterações neurológicas a sua progressão pode ser rápida e levar até a morte. $\mathrm{O}$ tratamento da AR é fundamentalmente médico. Os medicamentos e esquemas atuais poderiam prevenir ou retardar a aparição das alterações no nível atlanto-axial. $\mathrm{O}$ exame clínico e radiológico periódico permite detectar pacientes que já apresentem compromisso neurológico ou paciente com risco de desenvolver-lhe, sendo que em tais casos deve-se considerar a cirurgia como uma opção terapêutica. A recuperação neurológica pós-cirurgica depende do nível de compromisso neurológico prévio, o que reforça a importância da detecção e derivação rápida do paciente com risco. O tratamento cirúrgico deve fazer-se em centros especializados, por grupos multidisciplinares. Isto, junto com a intervenção rápida, ajudaria a reduzir as complicações peri-operatórias.

\section{ABSTRACT}

Rheumatoid arthritis affects millions of people all over the world. Up to 86\% of cases involve cervical spine alterations. Cervical spine instability patterns related torheumatoid arthritis are: atlanto-axial subluxation, basilar invagination and subaxial instability. Once neurological deficit develops, progression can be fast and even lead to death. Rheumatoid arthritis treatment is mainly nonsurgical. Current medication and treatment protocols may prevent or delay the development of atlanto-axial disease. Periodical clinical and radiological examination help diagnose patients who already have neurological symptoms or those who are at risk of developing them, and who should thus be considered for surgical treatment. Preoperative neurological deficit is a predictorofpostoperativeneurological recovery, thus the importance of early detection and referral of patients at risk. Surgical treatment should be performed in specialized centers, by multidisciplinary groups. Along with early intervention, this should help reduce perioperative complications.
DESCRITORES: Artrite reumatóide/ciurgia; Vértebras cervicais/patología; Doenças da coluna vertebral
KEYWORDS: Arthritis, rheumatoid/ surgery; Cervical vertebrae/ pathology Spinal diseases

\footnotetext{
'Staff member spine unit. Hospital Clínico Universidad de Chile, Servicio de Ortopedia y Traumatología. Santiago, Chile.

${ }^{2}$ Resident. Hospital Clínico Universidad de Chile, Servicio de Ortopedia y Traumatología. Santiago, Chile. 


\section{INTRODUCCIÓN}

$\mathrm{La}$ artritis reumatoidea (AR) es una enfermedad inflamatoria, crónica y sistémica, que afecta a las articulaciones sinoviales. La sinovitis y formación de pannus en dichas articulaciones conduce a una destrucción ósea con disfunción ligamentosa, la cual produce inestabilidad articular. En la columna cervical la AR puede afectar a las articulaciones atlanto-occipital, atlanto-axial, zigoapofisiarias y unco-vertebrales. Lo anterior provoca subluxación e inestabilidad, con diversos grados de compromiso medular, de las arterias vertebrales y riesgo de muerte súbita. Clásicamente se describe que entre un 17 y un $86 \%$ de los pacientes con $\mathrm{AR}$ presentan alteraciones a nivel cervical ${ }^{1}$. Sin embargo, una gran proporción de éstos no presenta manifestaciones neurológicas AL momento del exámen clínico, lo que puede llevar al médico tratante a pasar por alto lesiones importantes en la columna cervical. Durante las últimas décadas la AR aparentemente ha experimentado una disminución en su prevalencia ${ }^{2}$. Al mismo tiempo, la cantidad de pacientes con compromiso cervical en riesgo de compresión medular también es menor, lo que probablemente esté asociado al uso de nuevas drogas modificadoras anti-reumáticas $^{3}$. A pesar de lo anterior, las potenciales complicaciones que estos pacienets pueden presentar hacen que sea imprescindible conocer la historia natural de la enfermedad, sus manifestaciones clínicoradiológicas y las alternativas terapéuticas.

Las deformidades que pueden encontrarse en los pacientes con compromiso la columna cervical secundario a AR son:

1. Inestabilidad atlanto-axial (65\%). Provocada por la erosión del odontoides a nivel de su articulación con el arco anterior del atlas y por la elongación o ruptura del ligamento transverso. Estos cambios producen un desplazamiento anormal de $\mathrm{C} 1$ sobre $\mathrm{C} 2$ (con mayor frecuencia hacia anterior). Inicialmente éste puede ser reductible en extensión. Posteriormente, si se interpone pannus entre el arco del atlas y el odontoides, puede transformarse en una deformidad fija.

2. Impactación atlanto-axial (20\%) (también conocida como impactación basilar, invaginación pseudobasilar o subluxación vertical del axis). La destrucción de las articulaciones atlanto-axial, atlanto-ocipitales y de las masas laterales pueden provocar una impactación del atlas sobre el axis. Su pronóstico es peor debido al posible riesgo de compromiso del tronco encefálico.

3. Subluxación subaxial (15\%). La forma menos frecuente de la enfermedad a nivel cervical. Se presenta característicamente en distintos niveles, produciendo una deformidad "en escalera", con cifosis y estenosis multisegmentaria.

\section{Epidemiología e historia natural}

Entre el 40-80\% de pacientes con AR padece dolor cervical. El compromiso de la columna cervical ha sido reportado hasta en un $85 \%$ de los pacientes con $\mathrm{AR}^{1}$. Las alteraciones a nivel cervical aparecen temprano, generalmente dentro de los 2 primeros años luego del diagnóstico de la enfermedad ${ }^{4,5}$. El grado de daño en la columna cervical generalmente se correlaciona con la severidad de la enfermedad. Habitualmen-te éste se presenta en el contexto de una artritis periférica activa. La presencia de erosiones en las articulaciones de las manos y pies se asocia a subluxaciones a nivel cervical ${ }^{4,5}$.
El patrón radiológico más precoz es la inestabilidad atlanto-axial, que es inicialmente reductible. Con el avance de la enfermedad ésta se hace irreductible y, luego de seis años en promedio, aparece la impactación atlanto-axial ${ }^{6}$. En un estudio prospectivo de Pellici et al., el $80 \%$ de los pacientes mostró una progresión del compromiso radiológico, sin embargo sólo el 36\% tuvo progresión de los síntomas neurológicos ${ }^{7}$. En el mismo estudio, sólo el 50\% de los pacientes con inestabilidad radiológica presentaba síntomas neurológicos? ${ }^{7}$. De lo anterior se desprende que el grado de destrucción en la columna cervical, no siempre se corresponde con los síntomas, y que éste se detecta mediante el estudio radiológico.

$\mathrm{Si}$ bien la mayoría de las publicaciones corresponden a estudios retrospectivos, existen varias que sugieren que el curso natural de la enfermedad reumática a nivel cervical, especialmente en presencia de mielopatía, es de deterioro progresivo y riesgo de muerte súbita. Boden et al. evaluaron el curso clínico y la evolución radiológica de 73 pacientes por un período pro-medio de 7 años; 42 pacientes desarrollaron parálisis y 35 de ellos fueron tratados quirúrgicamente. De los 7 no intervenidos, el $100 \%$ presentó deterioro neurológico y 5 murieron por compresión de la médula espinal. En contraste, 25 de los 35 pacientes operados optimizaron su nivel ${ }^{8}$. Sunahara estudió la evolución de 21 pacientes tratados en forma conservadora; todos los pacientes quedaron postrados dentro de los tres años que siguieron al desarrollo de la mielopatía cervical y, después de 7 años todos habían fallecido (un tercio de ellos de manera abrupta, sin causa conocida) ${ }^{9}$. Mikulowski et al. realizó un estudio postmortem en 104 pacientes con AR encontrando 11 casos con subluxación atlanto-axial y compresión medular. Se documentó muerte súbita secundaria a eventos con hiperflexión en 7 de los 11 casos. En todos ellos la autopsia señaló, como causa más probable de muerte, la compresión medular por el odontoides ${ }^{10}$.

Una vez que aparecen alteraciones neurológicas, el deterioro puede progresar rápidamente. El $50 \%$ de los pacientes que desarrollanmieolpatíapuedenfallecerdentrodeunañosinosontratados ${ }^{11}$.

La morbimortalidad asociada a la AR cervical es multifactorial.La mielopatía puede ser producto de una compresión medular, por un evento isquémico determinado por la arteria espinal anterior, o la estenosis de los conductos para las arterias vertebrales ${ }^{12}$.

\section{Manifestaciones clínicas}

El examen clínico pude ser muy difícil de realizar e interpretar dada la presencia de otras manifestaciones neuromusculoesqueléticas. La atrofia muscular asociada al tratamiento esteroidal prolongado, las deformaciones articulares periféricas y la subluxación/ruptura de tendones pueden complicar la evaluación funcional de este grupo de pacientes.

Como fue expuesto anteriormente, el síntoma más frecuente es el dolor cervical. Ocasionalmente puede existir irradiación a la región occipital, cara y oídos. Estas se orinan por la compresión Del ramo pos-terior de $\mathrm{C} 2$ en pacientes con inestabilidad atlanto-axial.

Algunos de los pacientes pueden sentir un chasquido al extender el cuello, el que se produce por la reducción espontánea de la subluxación atlanto-axial. Este fenómeno es conocido como el signo de Sharp-Purser ${ }^{412}$. 
Los síntomas neurológicos pueden ser muy variados, y se presentan con menor frecuencia que el dolor. Dentro de los signos de compresión medular a nivel cervical (mielopatía cervical) podemos encontrar: una marcha espástica de base amplia, alteraciones del equilibrio y disminución de la habilidad motora fina en las manos. Se describe también la presencia de hiperreflexia, signos de Babinski y de Hoffmann, clonus y el reflejo braquio-radial invertido, entre otros ${ }^{2}$. El signo de "L'Hermitte" que es la sensación de electricidad en la región del dorso y extremidades, como consecuencia del movimiento del cuello en una columna inestable, es otro hallazgo que sugiere la prescencia de compresión medular. En forma tardía, también pueden observarse retención urinaria y distintos grados de debilidad muscular en las extremidades ${ }^{2,12}$.

Los síntomas asociados a la insuficiencia vertebrobasilar por la impactación atlanto-axial incluyen: lipotimias, vértigo, disfagia, convulsiones, tinitus, alteraciones en el equilibrio, disartria, nistagmus o desórdenes visuales ${ }^{13}$.

\section{Imagenología}

Radiología convencional - Se deben solicitar radiografías cervicales a todos los pacientes AR que presenten ${ }^{12}$ :

1. Sintomatología cervical por más de 6 meses; 2 . Síntomas o signos neurológicos; 3 . Procedimientos programados que requieran intubación endotraqueal en pacientes que no han sido evaluados radiográficamente en los últimos 2-3 años; 4. Destrucción ósea, rápidamente progresiva, del carpo o tarso; 5. Deterioro funcional que progresa rápidamente.

La evaluación imagenológica básica incluye radiografías laterales, anteroposterior y dinámicas, además de la radiografía de C1-C2 transoral. Varias mediciones pueden aplicarse a las imágenes para evaluar las distintas alteraciones a nivel cervical. La distancia atlantodental anterior (AADI) es medida en una radiografía lateral desde la cara anterior del odontoides hasta la cara posterior del arco anterior del atlas. Tradicionalmente se ha considerado que una distancia mayor a $5 \mathrm{~mm}$ hace el diagnóstico de subluxación atlanto-axial ${ }^{2,12}$. Actualmente esta medida ha perdido importancia debido a que no siempre es fácil de visualizar y no se correlaciona con el riesgo de lesión neurológica.

La distancia atlantodental posterior (PADI) es la distancia medida entre la superficie posterior del odontoides y la cara anterior del arco posterior de $\mathrm{C} 1$ en una radiografia lateral (Figura 1).

El diámetro del canal subaxial se mide de manera similar en la columna caudal al axis, donde el odontoides es reemplazado por el cuerpo vertebral de la columna cervical.

Existe una variedad importante de líneas que han sido utilizadas para medir la impactación basilar (McGregor, McRae, Chamberlain, Wackenheim, Fischgold-Metzger, etc.). La línea de McGregor se traza en una radiografía lateral, desde el borde posterior del paladar duro hasta el punto más caudal y externo de la concha del occipital. La impactación atlanto-axial ocurre cuando el borde superior del odontoides supera los 4,5 mm sobre esta línea.14 La desventaja de esta medición es la difícil definición de los aspectos óseos a determinar, especialmente cuando existe erosión de la apófisis odontoides (Figura 2A).

Otras técnicas permiten diagnosticar la impactación -axial a pesar de la presencia de erosión de la odontoides. Estas son la medición de Redlund-Johnell, el índice de Ranawat y las estaciones de Clark. La medición de Redlund-Johnell se realiza en una radiografía lateral y se calcula como la distancia entre la línea de McGregor y el punto medio del margen inferior del cuerpo del axis. Valores menores a $34 \mathrm{~mm}$ en el hombre y
$29 \mathrm{~mm}$ en la mujer son indicadores de invaginación basilar ${ }^{14}$ (Figura 2B). El índice de Ranawat mide la distancia entre el centro del pedículo de $\mathrm{C} 2$ y el eje transverso del atlas, a través del eje axial del odontoides. Se consideran alterados los valores menores a $15 \mathrm{~mm}$ en el hombre y $13 \mathrm{~mm}$ em mujeres ${ }^{14}$ (Figura 3). La estación de Clark se determina dividiendo al odontoides en 3 partes iguales en el plano sagital. Se está en prescencia de una invaginación basilar si el arco anterior del atlas se encuentra al mismo nivel que el tercio medio (segunda estación) o el tercio nferior (tercera estación) del odontoides ${ }^{14}$.

Ningún examen demuestra la presencia de déficit neurológico, sólo tienen por función determinar las poblaciones en riesgo. Los predictores de riesgo de déficit neurológico más importantes en las radiografías son el PADI y el diámetro subaxial.

Boden et al., estableció que un PADI menor de $14 \mathrm{~mm}$ se correlaciona con el desarrollo de parálisis ${ }^{8}$. Cabe recordar que el PADI no representa el espacio libre para la médula, dado que el pannus no es visible en la radiografía, por lo que pacientes con valores superiores a $14 \mathrm{~mm}$ pueden presentar compresión medular (Figura 4).

El PADI pre-operatorio también puede ser utilizado para predecir la recuperabilidad de los pacientes que serán operados. En el mismo trabajo, Boden describió que los pacientes con un PADI menor a $10 \mathrm{~mm}$ presentaban escasa recuperación neurológica. En su estudio, todos aquéllos en que el PADI era mayor a $10 \mathrm{~mm}$ presentaron algún grado de recuperación. En aquellos que además tenían una impactación atlanto-axial, solo mejoraron

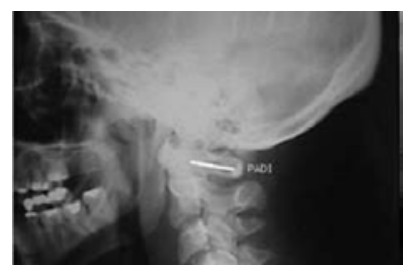

Figura 1

Distancia atlantodental posterior (PADI)
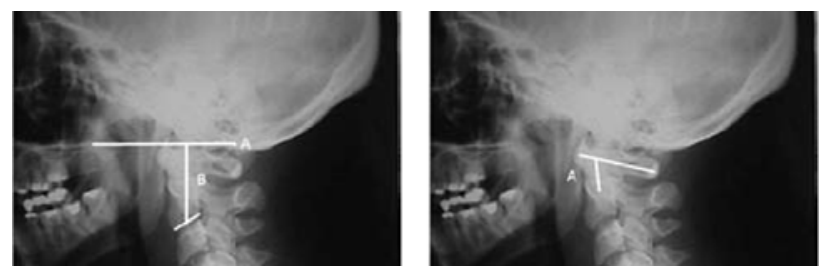

Figura 2

A) Línea de McGregor.

B) Método de Redlund-Johnell

Figura 3

A) Método de Ranawat

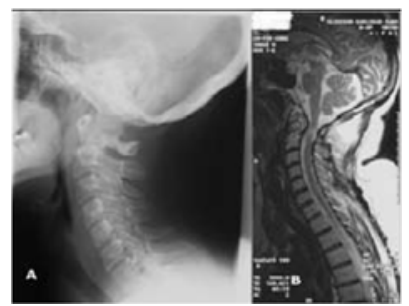

Figura 4

A) Radiografía lateral con un PADI normal. B) Resonancia Magnética del mismo pacinte que demuestra compresión medular por el pannus detrás de la odontoides. (Foto cortesía Dr. R.P. Melcher)

neurológicamente quienes tenían un PADI de $13 \mathrm{~mm}$ o más ${ }^{8}$.

Un diámetro sagital subaxial menor de $14 \mathrm{~mm}$ se correlaciona con alteraciones neurológicas en el exámen clínico y es por tanto considerado crítico en los pacientes con compromiso reumático ${ }^{8}$.

En relación a la impactación atlanto-axial, Riew et al. estudiaron las distintas medidas disponibles; concluyendo que éstas tienen baja sensibilidad, especificidad y no son capaces de 
predecir el compromiso neurológico por si sólas. No obstante, la combinación de los criterios de Redlund-Johnell, Ranawat y Clark, tendrían una sensibilidad de $94 \%$ y un valor predictivo negativo de $91 \%{ }^{14}$.

Tomografía computada (TAC) - La TAC ayuda a identificar los detalles anatómicos, demostrando la extensión de las erosiones, siendo las reconstrucciones sagitales y coronales las que otorgan la mayor ayuda. Es importante solicitar cortes finos (1 a $2 \mathrm{~mm}$ ) para obtener los mejores beneficios de este examen. Una de sus desventajas es que las imágenes se adquieren con el paciente en decúbito, posición en la que las deformidades pudieran reducirse ${ }^{2}$. Se le considera actualmente una herramienta útil en la planificación pre-operatoria.

El uso de contraste intratecal puede demostrar áreas de compresión medular, pero el rol de la mieloTAC ha sido des plazado por la resonancia magnética (RM), siendo su mayor utilidad en aquellos pacientes con contraindicación de RM.

Resonancia magnética - La RM es extremadamente útil en la identificación de la compresión medular en la AR cervical al definir si son elementos óseos o inflamatorios (pannus) los que provocan la estenosis. El aumento de señal en las imágenes en T2 son sugerentes de mielomalacia, gliosis o edema y se han asociado a una menor recuperación neurológica postquirúrgi$\mathrm{ca}^{12}$. Este examen permite la obtención de imágenes dinámicas, logrando visualizar componentes funcionales en la inestabilidad y compresión.

Una de las mediciones que sugieren la presencia de compresión medular es el ángulo cérvicomedular, descrito por Bundschuh et al. ${ }^{15}$, el cuál se calcula en una imagen medio sagital. El ángulo se traza uniendo una línea paralela al eje de la médula espinal, y otra paralela al eje del bulbo raquídeo. El valor normal se encuentra entre los $135^{\circ}$ y $175^{\circ}$. Un ángulo menor a $135^{\circ}$ es señal de compresión medular con evidencia clínica de mielopatía ${ }^{15}$.

Se ha planteado también el uso de la RM dinámica en la evaluación de los pacientes. Dvorak et al. reportó que los pacientes cuyo diámetro medular menor de $6 \mathrm{~mm}$ en flexion estan en riesgo de déficit neurológico ${ }^{16}$. Sin embargo, debido a al riesgo teóricode muerte súbita en pacientes con inestabilidad severa, su uso rutinario es cuestionado ${ }^{12}$.

\section{Tratamiento}

Los objetivos del tratamiento en estos pacientes son: aliviar el dolor y evitar el desarrollo de déficit neurológico irreversible y muerte súbita. El tratamiento conservador incluye el uso de drogas anti-reumáticas, terapia física y el uso de órtesis. Debe ser iniciado precozmente y siempre acompañarse del seguimiento clínico y radiológico para detectar a tiempo la progresión de la enfermedad. Con esta modalidad de tratamiento es posible conseguir alivio del dolor a largo plazo ${ }^{17}$. Frente a la presencia de compromiso radiológico progresivo, o deterioro neurológico, se debe considerar el tratamiento quirúrgico2,11. Adicionalmente, existen estudios que sugieren que, el tratamiento precoz con drogas antirreumáticas modificadoras de la enfermedad es capaz de prevenir o retardar el desarrollo de alteraciones a nivel atlanto-axial ${ }^{18,19}$.

Las indicaciones quirúrgicas incluyen: presencia de inestabilidad, déficit neurológico y dolor cervical intratable ${ }^{2,11}$ Frente a la presencia de déficit neurológico o de dolor intratable, la indicación quirúrgica es clara. Las dificultades se presentan en lo pacientes que, teniendo inestabilidad radiológica, no tienen alteraciones neurológicas ni dolor importante. Algunos autores proponen que el manejo quirúrgico precoz puede ayudar a prevenir la progresión de la enfermedad; en manos expertas, la inestabilidad, deformidad y dolor asociados a la enfermedad a nivel cervical podrían ser tratados en forma programada, con baja mortalidad y morbilidad asociadas ${ }^{3}$.

Basándose en el riesgo de parálisis observado en su estudio, Boden et al. propuso utilizar los siguientes parámetros radiológicos para indicar la cirugía en presencia o ausencia de síntomas neurológicos ${ }^{8}: 1$. Inestabilidad atlanto-axial con PADI igual o $<14 \mathrm{~mm}$; 2. Migración del odontoides sobre la línea de Mc Gregor > o igual a 5mm y 3) un diámetro sagital del canal medular igual o $<$ a $14 \mathrm{~mm}$, en presencia de subluxación subaxial. A estos criterios pudiera agregarse la combinación de la alteración de las mediciones de Ranawat, Redlund-Johnell y la estación de Clark ${ }^{14}$. Por último, también debe considerarse un ángulo cervicomedular $<135^{\circ}$ en la $\mathrm{RM}^{15}$ o evidencias de compresión medular (en RM o TAC) 2,12. $^{2}$.

Si la cirugía es indicada, el objetivo del tratamiento incluye la reducción de la subluxación, descompresión del tejido neural comprometido y la estabilización para mantener el alineamiento y aliviar el dolor.

En el momento de la cirugía, la intubación bajo visión directa, con ayuda de fibrobroncoscopía y paciente vigil, minimizan los riesgos y optimizan el control del movimiento cervical ${ }^{12}$.

La subluxación atlanto-axial reductible puede ser manejada con una fusión C1-C2. Para ello existen una variedad de técnicas, entre ellas la de Harms, Magerl y Gallie. Si existe irreductibilidad de esta alteración, o una reducción parcial del canal, se hace necesaria una descompresión (laminectomía de C1).

En los casos de impactación atlanto-axial se recomienda el uso pre-operatorio de tracción, mediante un halo, para intentar reducir la impactación y evitar la necesidad de realizar una descompresión. Si la tracción es exitosa puede llevarse a cabo una instrumentación y artrodesis posterior de $\mathrm{C} 0$ a $\mathrm{C} 2$. Las imágenes preoperatorias indican si la fusión debe ser prolongada hacia caudal, incluyendo todos los segmentos subluxados. Si La impactacion atlanto-axial es irreductible o, una fusión posterior sin reducción persiste sintomática, la odontoidectomía está indicada, pudiendo realizarse por vía transoral o con un abordaje retrofaringeo alto.

La inestabilidad subaxial puede ser tratada mediante técnicas de fusión y descompresión por via posterior o anterior. La fusión con descompresión son técnicas que han sido utilizadas con éxito para mejorar el dolor cervical en los pacientes con AR.2 Además, el pannus presente en las articulaciones, que participa en la compresión medular desaparece una vez lograda la estabilización (Figura 5). Sin embargo, los resultados de los procedimientos quirúrgicos son más variables en relación a la recuperación neurológica. Uno de los predictores de recuperación post operatoria más importante es la condici-

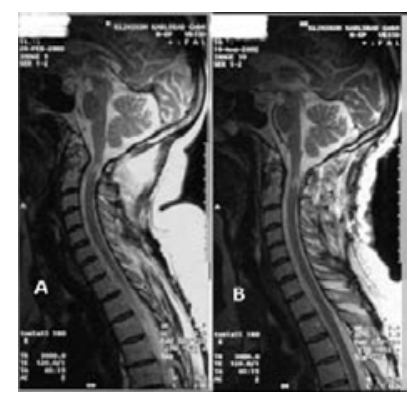

Figura 5

A) Resonancia magnética pre-operatoria. B) RM 1 mes post-operatoria en la que se observa reabsorción del pannus. (Foto cortesía Dr R.P. Melcher) 
ón neurológica previa ${ }^{2,12}$. Mientras mayor sea el deterioro preoperatorio, menor es la recuperación esperada. Esto último no hace más que reforzar la importancia de un diagnóstico precoz, y de la cirugía como herramienta que debe ser considerada antes de que ocurra un deterioro neurológico importante.

\section{Complicaciones}

El tratamiento quirúrgico de la AR cervical se ha asociado a un alta incidencia de complicaciones. La mortalidad perioperatoria descrita alcanza hasta el 10\%.12 Sin embargo, estudios más recientes muestran una disminución importante de la mortalidad y las complicaciones quirúrgicas, probablemente gracias a la detección y derivación oportuna de los pacientes con compromiso neurológico ${ }^{11}$. También puede agregarse el tratamiento en centros especializados, con equipos multidisciplinarios y con mayor experiencia en el tratamiento de pacientes con $\mathrm{AR}^{3}$.

Las complicaciones incluyen infección, dehisencia de sutura y pérdida de la reducción. La incidencia de pseudoartrosis ha sido reportada en la literatura en rangos que van de $0 \%$ a $50 \%$. También se ha descrito la presencia de descompensación del seg- mento adyacente hasta en el $15 \%$ de los pacientes ${ }^{20}$. Esta última se observaría con mayor frecuencia luego de fijaciones cráneocervicales, especialmente en aquellos paciente con alteraciones pre-operatorias significativas en la columna cervical sub-axial ${ }^{11}$.

\section{Conclusiones}

La artritis reumatoidea es una enfermedad que afecta a un número importante de personas a nivel mundial. Aproxima-damente entre un $10 \%$ de ellos pueden ser candidatos a una estabilización quirúrgica cervical.

Es necesario detectar a los pacientes con artritis reumatoidea que presentan un riesgo inaceptable de déficit neurológico irreversible o fatal. La evaluación seriada, junto a un examen neurológico cuidadoso y al seguimiento radiográfico, son las principales herramientas disponibles para su evaluación.

La derivación oportuna de aquellos pacientes que requieren cirugía es fundamental para lograr un mejor pronóstico de recuperación neurológica. El manejo de estos casos debe ser llevado a cabo por equipos multidisciplinarios, con experiencia en el tratamiento de pacientes con AR.

\section{REFERÊNCIAS}

1. Rawlins BA, Girardi FP, Boachie-Adjei O. Rheumatoid arthritis of the cervical spine. Rheum Dis Clin North Am. 1998;24(1):55-65.

2. Lee MJ, Bransford RJ. Spinal manifestations of rheumatoid arthritis. Contemp Spine Surg. 2008;9(11): 1-9.

3. Choi D, Casey ATH, Crockard HA. Neck problems in rheumatoid arthrits - changing disease patterns, surgical treatments and patient's expectations. Rheumatology. 2006;45(10):1183- 4.

4. Winfeld J, Young A, Williams P, Corbett M.: Prospective study of the radiological changes in hands, feet, and cervical spine in adult rehumatoid disease. Ann Rheum Dis 1983;42(6):613-8.

5. Paimela L, Laasonen L, Kankaanpää E, Leirisalo-Repo M.: Progression of cervical spine changes in patients with early rheumatoid arthritis. J Rheumatol. 1997;24(7):1280-4.

6. Oda T, Fujiwara K, Yonenobu K, Azuma B, Ochi T. Natural course of cervical spine lesions in rheumatoid arthritis. Spine. 1995; 20(10):1128-35.

7. Pellicci PM, Ranawat CS, Tsairis $P$, Bryan WJ.: A prospective study of the progression of rheumatoid arthritis of the cervical spine. J Bone Joint Surg Am. 1981; 63(3):342-50.

8. Boden SD, Dodge LD, Bohlman HH, Rechtine GR. Rheumatoid arthritis of the cervical spine. Along-term analisys with predictors of paralysis and recovery. $\mathrm{J}$ Bone Joint Surg Am. 1993; 75(9):128297.

9. Sunahara N, Matsunaga S, Mori T, Ijiri K, Sakou T. Clinical course of conservatively managed rheumatoid arthritis patients with myelopathy . Spine. 1997;22(22):2603-8.
10.Mikulowski P, Wollheim FA, Rotmil P, Olsen I.: Sudden death in rheumatoid arthritis with atlanto-axial dislocation. Acta Med Scand. 1975;198(6): 445-51.

11. Casey AT, Crockard HA, Pringle J, O'Brien MF, Stevens JM. Rheumatoid arthritis of the cervical spine: current techniques for management. Orthop Clin N Am 2002;33:291-309.

12. Kim DH, Hilibrand AS. Rheumatoid arthritis in the cervical spine. J Am Acad Orthop Surg . 2005;13(7):463-74.

13. Dreyer S, Boden S. Natural history of rheumatoid arthritis of the cervical spine. Clin Orthop. 1999;366: 98-106.

14. Riew KD, Hilibrand AS, Palumbo MA, Sethi N, Bohlman HH. Diagnosing basilar invagination in rheumatoid patient: The reliability of radiographic criteria. J Bone Joint Surg Am. 2001;83(2):194-200.

15.Bundschuh C, Modic MT, Kearney F, Morris R, Deal C. Rheumatoid arthritis of the cervical spine: Surfacecoil MR imaging. Am J Roentgenol. 1988;151(1):181-7.

16.Dvorak J, Grob D, Baumgarter H, Gschwend N, Grauer W, Larsson S. Functional evaluation of the spinal cord by magnetic resonance imaging in patients with rheumatoid arthritis and inestability of upper cervical spine. Spine 1989;14(10):1057-64.

17. Kauppi M, Leppänen L, Heikkilä S, Lahtinen T, Kautiainen H. Active conservative treatment of atlantoaxial subluxation in rheumatoid arthritis. Br J Rheumatol 1998;37(4):417-20.
18. Neva MH, Kauppi MJ, Kautiainen H, Luukkainen R, Hannonen P, LeirisaloRepo M, et al. Combination drug therapy retards the development of rheumatoid atlantoaxial subluxations. Arthritis Rheum. 2000;43(11):2397-401.

19. Kauppi MJ, Neva MH, Lahio K, Kautiainen H, Luukkainen R, Karjalainen A, et al. Rheumatoid atlantoaxial subluxation can be prevented by intensive use of traditional disease modifying antirheumatic drugs. J Rheumatol. 2009;36(2):273-8.

20. Agarwal AK, Peppelman WC, Kraus DR, Pollock BH, Stolzer BR, Eisenbeis $\mathrm{CH}$ Jr, et al. Recurrence of cervical spine instability in rheumatoid arthritis following previous fusion: can disease progression be prevented by early surgery?. J Rheumatol. 1992;19(9):136470. 